\title{
Modulating the gut-liver axis and the pivotal role of the faecal microbiome in cirrhosis
}

\author{
Authors: Charlotte Woodhouse, ${ }^{A}$ Arjuna Singanayagam ${ }^{B}$ and Vishal $C$ Patel ${ }^{C}$
}

Cirrhosis is associated with intestinal dysbiosis, with specific alterations in the gut microbiota linked to particular aetiologies and manifestations of the disease. We review the role of the gut microbiome and the importance of the intestinal barrier in cirrhosis, provide an overview of the terminology and techniques relevant to this emerging area, and discuss the latest developments in therapies targeting the gut-liver axis.

\section{Introduction}

The human gut harbours trillions of micro-organisms that in health coexist symbiotically with their host. The gut microbiota (the micro-organisms in the intestinal niche), also referred to as the microbiome (the micro-organisms and their collective genomes), consists of bacteria, archaea, fungi, viruses and protozoa. ${ }^{1}$ This collection of microbes perform a multitude of functions benefiting the host, which have become better understood over the past decade, in part due to the advent of next-generation sequencing technologies.

Within the gut microbiota, bacteria are the most studied owing to the recent advent of non-culture-based molecular techniques, such as $16 \mathrm{~S}$ ribosomal ribonucleic acid (rRNA) gene sequencing and shotgun metagenomic profiling, that allow characterisation of bacteria, and with the latter technique characterisation of their potential functions, without having to grow them all in a laboratory. $16 \mathrm{~S}$ sequencing amplifies this highly conserved 1,500 base pair gene (found in all bacteria and archaea) to allow genus-level identification. This has largely been superseded by metagenomic approaches which sequence all of the deoxyribonucleic acid (DNA) in a sample. Metagenomic approaches provide much higher phylogenetic resolution, allowing species-level identification, and can also provide information on bacterial gene function. Other techniques such as transcriptomics

Authors: A hepatology clinical research fellow, King's College London, London, UK and King's College Hospital NHS Foundation Trust, London, UK; ${ }^{\text {B }}$ consultant hepatologist, St George's University Hospitals NHS Foundation Trust, London, UK and senior clinical lecturer, St George's University of London, London, UK; ' ${ }^{\text {consultant }}$ hepatologist, King's College Hospital NHS Foundation Trust, London, UK, honorary senior lecturer, King's College London, London, UK and principal investigator, Institute of Hepatology, Foundation for Liver Research, London, UK and metabonomics provide information on the genes that are transcribed and thus active and their metabolic products, respectively. These various analytical approaches are summarised in Fig 1 and the related terminology is outlined in Table 1.

\section{Gut microbiota in health}

The Human Microbiome Project examined microbial populations at 15 body sites in men and 18 in women, providing a reference data set from 242 healthy Americans. ${ }^{2}$ This showed that microbial populations vary from individual to individual, but all perform similar functions and exhibit diversity. The majority of human gut bacteria are from the Bacteroidetes and Firmicutes phyla. There is also a minor contribution from Actinobacteria, Verrucomicrobia

\section{Key points}

Patients with cirrhosis are predisposed to small intestinal bacterial overgrowth (SIBO), altered gut permeability and dysbiosis, resulting in pathological bacterial translocation (BT) which drives systemic inflammation.

Cirrhotic patients have an over-representation of potential pathogens in their gut microbiota, such as the Gram-negative Enterobacteriaceae family which includes Escherichia coli and other bacterial species. This can lead to infections including spontaneous bacterial peritonitis and contribute to acute hepatic decompensation.

Manipulation of the gut microbiota by antibiotics, pre-/ probiotics, faecal microbiota transplantation or stool transplantation (FMT) as well as other pharmacological approaches can ameliorate gut microbial dysbiosis, induce 'rebiosis' and potentially reduce the risk of decompensation.

FMT has shown promise in small-scale human studies and now requires larger studies with long-term follow up and rigorous safety controls to further evaluate clinical efficacy.

KEYWORDS: Microbiota, cirrhosis, gut-liver axis, dysbiosis, faecal microbial transplantation

DOI: 10.7861/clinmed.2020-0676 
Current techniques in human microbiome profiling

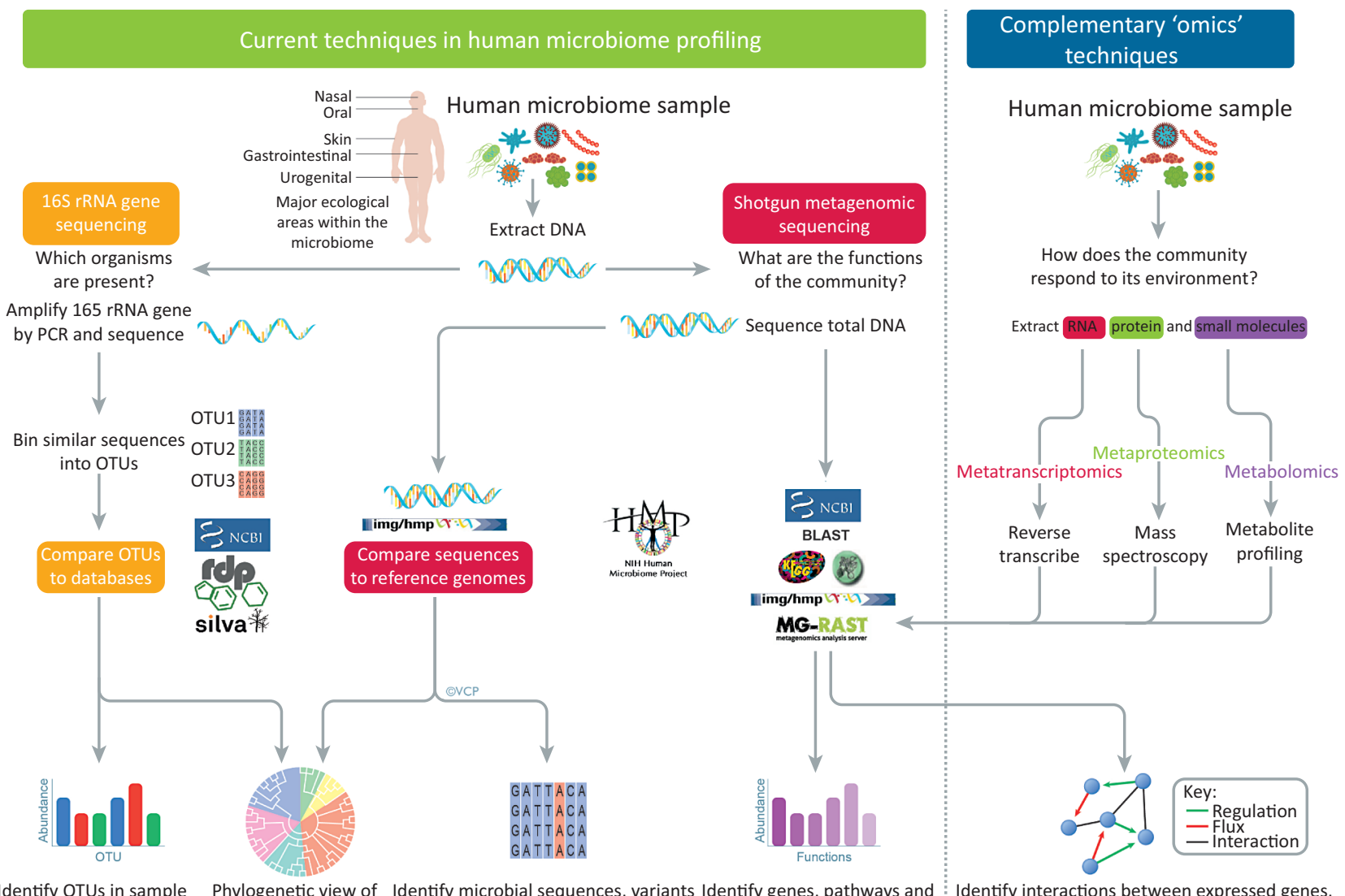

Identify OTUs in sample Phylogenetic view of Identify microbial sequences, variants Identify genes, pathways and Identify interactions between expressed genes, and relative frequencies community composition and polymorphisms in sample relative frequencies in sample : proteins and metabolic products in sample

Fig 1. Overview of next generation sequencing and bioinformatic methods for microbiome analyses and functional metagenomics. DNA = deoxyribonucleic acid; OTU = operational taxonomic unit; PCR = polymerase chain reaction; rRNA = ribosomal ribonucleic acid.

and Proteobacteria as well as archaea (methane producing microorganisms) and bacteriophages (viruses which infect bacteria). Gut bacteria produce short-chain fatty acids (SCFAs) from otherwise indigestible starches such as butyrate, which plays a major role in maintaining gut barrier integrity, and acetate, which is involved in cholesterol synthesis. The microbiota also contributes to host energy production, vitamin synthesis, drug metabolism and bile acid metabolism and plays a vital role in host immune system maturation. $^{3}$

Bile acids modulate the gut microbiota via their antimicrobial activity and control its composition based on bacterial enzyme activity. Primary bile acids are produced from cholesterol in the liver and bile acids re-circulate via the enterohepatic circulation, with $95 \%$ re-absorbed in the terminal ileum. Bile acids that reach the colon are metabolised by two types of bacterial enzymes. Bile salt hydrolases (BSHs), which are expressed by a variety of different bacteria, deconjugate glycine and taurine, reforming primary bile acids. $7 \alpha$-hydroxylation converts them to secondary bile acids. ${ }^{3}$ Disruption to this finely balanced process can occur due to altered bile acid production as seen in cirrhosis, where there is reduced conversion of primary to secondary bile acids, or altered bacterial composition. ${ }^{4}$

The gut microbiota is also influenced by route of delivery at birth (vaginal or caesarean section), breast or formula feeding, diet, drugs and alcohol in later life, as well as a whole variety of other environmental factors that remain to be determined. There is growing interest in perturbations of the normal gut microbiome seen in various disease states, such as diabetes mellitus, inflammatory bowel and cardiovascular diseases, and Alzheimer's disease. This is commonly and very broadly described as 'dysbiosis' and the impact of these compositional changes on microbiome function and host interactions is where research is increasingly focused. Dysbiosis refers to several types of changes including: ${ }^{5}$

> a general change in microbiota composition, eg alteration, perturbation, abnormal composition or loss of diversity

$>$ an imbalance in composition, which is almost always deemed to have a negative effects

> changes to specific taxonomic lineages in that composition.

The intestinal barrier relies on multiple layers that defend against exogenous pathogens. ${ }^{6}$ In the normal homeostatic state, the most external layer of defence is mucus, which is where the outer, microbiota-colonised layer interacts directly with the gut microbiota, while the inner sterile layer covers the gut epithelium. ${ }^{7}$ This epithelial monolayer of cells maintains a physical barrier which is dependent on dynamic tight junctions to maintain its integrity. ${ }^{8}$ The next layer of defence is provided by the immune cells of the lamina propria, which contains several types of innate and adaptive immune cells and aggregations of lymphoid nodules known as Peyer's patches. ${ }^{9,10}$ The final layer is 
Table 1. Terminology and definitions relevant to microbiome descriptions and techniques

Term

Next generation

sequencing

16S rRNA

16S rRNA gene

sequencing

Metagenomics

Functional metagenomics

Microbe

Microbiota

Microbiome

Meta-transcriptomics

Meta-proteomics

Metabolomics

Metabonomics

Diversity

Dysbiosis

Prebiotic

Probiotic

Taxonomy

Taxon

\section{Definition}

Encompasses a broad range of sequencing technologies which require preparation of templates (recombinant DNA combining adaptor with the target sequences), sequencing, genome alignment and assembly. These methodologies may be run in parallel on a large scale ('massively parallel'), making their sequencing affordable and more efficient than the 'first generation' Sanger sequencing technology

The transcribed form of the $16 \mathrm{~S}$ ribosomal subunit gene, the smaller RNA component of the prokaryotic ribosome, used as the most common taxonomic marker for bacterial communities

Sequencing of the highly conserved 1,500 bp gene found in bacteria and archaea allowing genus level resolution (cheaper and faster than metagenomics)

Obtained via shotgun sequencing of all the DNA in a sample, followed by mapping to a reference database. The metagenome is the collection of genomes and genes from members of the microbiota

Computational or experimental analysis of a microbial community with respect to the biochemical and other biomolecular activities encoded by its composite genome

A microorganism of the Bacteria, Archaea or Eukarya life domains and their viruses

The total collection of microbial organisms within a community, typically used in reference to a mammalian host and to particular anatomical niches, eg the gastrointestinal tract

The total microbial community, their genomes and biomolecules within a defined environment Analysis of the expressed RNA by high-throughput sequencing

Sequencing of all the proteins present in a given sample (from the micro-organisms and the surrounding environment)

Analysis of the metabolite profile in a sample (the collective metabolites are termed the metabolome)

Describes the approach to generate metabolite profiles from complex systems such as urine, faecal water (eg where more than one strain or tissue has generated the metabolites)

A calculated index of the taxonomic distribution within a community, either in terms of distinct taxa or in terms of their evolutionary/phylogenetic distance, that incorporates measures of richness and species distribution

Imbalance between microbes among a community that occupies a given ecological niche. The concept of dysbiosis was coined by Metchnikoff and describes a disordered commensal community that alters homeostasis between the microbiota and its host, predisposing to risk of or contributing to disease

A food substance metabolised by the microbiota so as to directly or indirectly benefit the host

A live microorganism that, when ingested, provides benefit to the host, either directly through interactions with host cells or indirectly through effects on members of the microbiota

Discipline of classification for organisms, formally described by Carl Linnaeus (1707-1778). Current classification systems use a series of tiers starting from domain, and progressing to kingdom, phylum, class, order, family, genus, species and strain

A taxonomic unit or population of organisms, whether named or un-named, which are inferred to be related and have common characteristics

$\mathrm{bp}=$ base pairs; DNA = deoxyribonucleic acid; rRNA = ribosomal ribonucleic acid

the gut vascular barrier (GVB), which is pivotal in preventing the systemic dissemination of viable microbes and their metabolites via the portal circulation. ${ }^{11}$ The gut microbiota play an important role in interacting homeostatically with all these various layers, in particular in the priming of gut mucosal immunity. ${ }^{12,13}$

\section{Gut microbiota in cirrhosis}

The composition of the dysbiotic gut microbiota in cirrhosis has been characterised by several groups, describing a clear over-representation of pathogenic bacteria and fungi. Qin et al evaluated the gut microbiome of 98 Chinese cirrhotic subjects and compared them to 83 healthy controls by shotgun metagenomic sequencing. ${ }^{14}$ Veillonella, Streptococcus, Clostridium and Prevotella were enriched in the cirrhosis cohort, with a dominance of Eubacterium and Alistipes in the healthy control group. Of the 20 species that were enriched in cirrhosis, four were Streptococcus species and six were Veillonella species, including species typically originating from the oral cavity, suggesting that these two genera may have an important role in cirrhosis and leading to the concept of 'oralisation' of the gut microbiome in cirrhosis. In a study of 244 cirrhotic subjects with varying severity of disease and 25 age-matched controls, the term 'cirrhosis dysbiosis ratio (CDR)' was developed, which compares the ratio of beneficial to potentially pathogenic bacteria, with a low number being indicative of dysbiosis. ${ }^{15}$ The 
CDR was found the be highest in controls and to reduce with increasing severity of disease, being lowest in cirrhotic inpatients with acute hepatic decompensation. CDR also negatively correlated with endotoxaemia. There was a significant reduction in autochthonous taxa (Clostridiales XIV, Ruminococcaceae and Lachnospiraceae) and a significant increase in pathogenic taxa (Enterococcaceae, Staphylococcaceae, Enterobacteriaceae) when comparing controls with cirrhotic outpatients and inpatients. A reduction in Veillonellaceae and Porphyromonadaceae with worsening liver disease was also noted.

Changes to the fungal inhabitants of the gut microbiota have also been reported in alcohol-related cirrhosis. Patients with alcoholic liver disease have reduced gut fungal diversity and overgrowth of Candida. ${ }^{16}$ These patients also have increased $\beta$-glucan in the systemic circulation (derived from fungal cell walls) which was ameliorated by anti-fungal treatment in a mouse model. Children with non-alcoholic steatohepatitis (NASH) have been found to have an over-representation of ethanol-producing bacteria, suggesting that the pathogenesis of NASH may relate to endogenous ethanol production, resulting in liver damage. ${ }^{17}$ Specific gut microbiota changes have also been noted in primary sclerosing cholangitis (PSC) with an increase in Veillonella, and gut microbiota profiles are distinct from those with inflammatory bowel disease without PSC and healthy controls. ${ }^{18}$

There is increasing evidence that gut dysbiosis in cirrhosis can pathologically contribute to disease progression and decompensating events such as spontaneous bacterial peritonitis (SBP) and hepatic encephalopathy (HE). Patients with cirrhosis often develop small intestinal bacterial overgrowth (SIBO) which is reported to be more common in patients with advanced cirrhosis. SIBO (as measured by hydrogen breath testing) occurred in $20 \%$ of Child-Pugh A cirrhotics, compared to $73 \%$ of those with Child-Pugh C, but only $8 \%$ of healthy controls, and has been associated with development of SBP. ${ }^{19}$ Chang et al showed that $70 \%$ of cirrhotic patients with SBP had SIBO, compared to $20 \%$ of those without SBP. Small intestinal motility was also noted to be impaired in those with a history of SBP and may contribute to the development of SIBO. ${ }^{20}$ An important consideration is the effect of pharmacotherapies that cirrhotic patients are often prescribed, which can have a deleterious effect on the gut microbiota. Proton pump inhibitors (PPIs) are an example of a commonly used medication that is associated with poor outcomes in cirrhosis, being linked to increasing risk of infections and HE. ${ }^{21}$ PPI use has been shown to be associated with a relative enrichment in the distal gut of microbiota that are usually resident in the oral cavity in cirrhotics. ${ }^{22}$ This has deleterious functional consequences whereby Streptococcaceae - known to increase urease generation - are enriched along with greater production of trimethylamine $\mathrm{N}$-oxide. ${ }^{23}$

Patients with cirrhosis exhibit increased gut permeability, facilitating pathological translocation of bacteria and their immune-interacting products (known as pathogen-associated molecular patterns or PAMPs) into the systemic circulation,

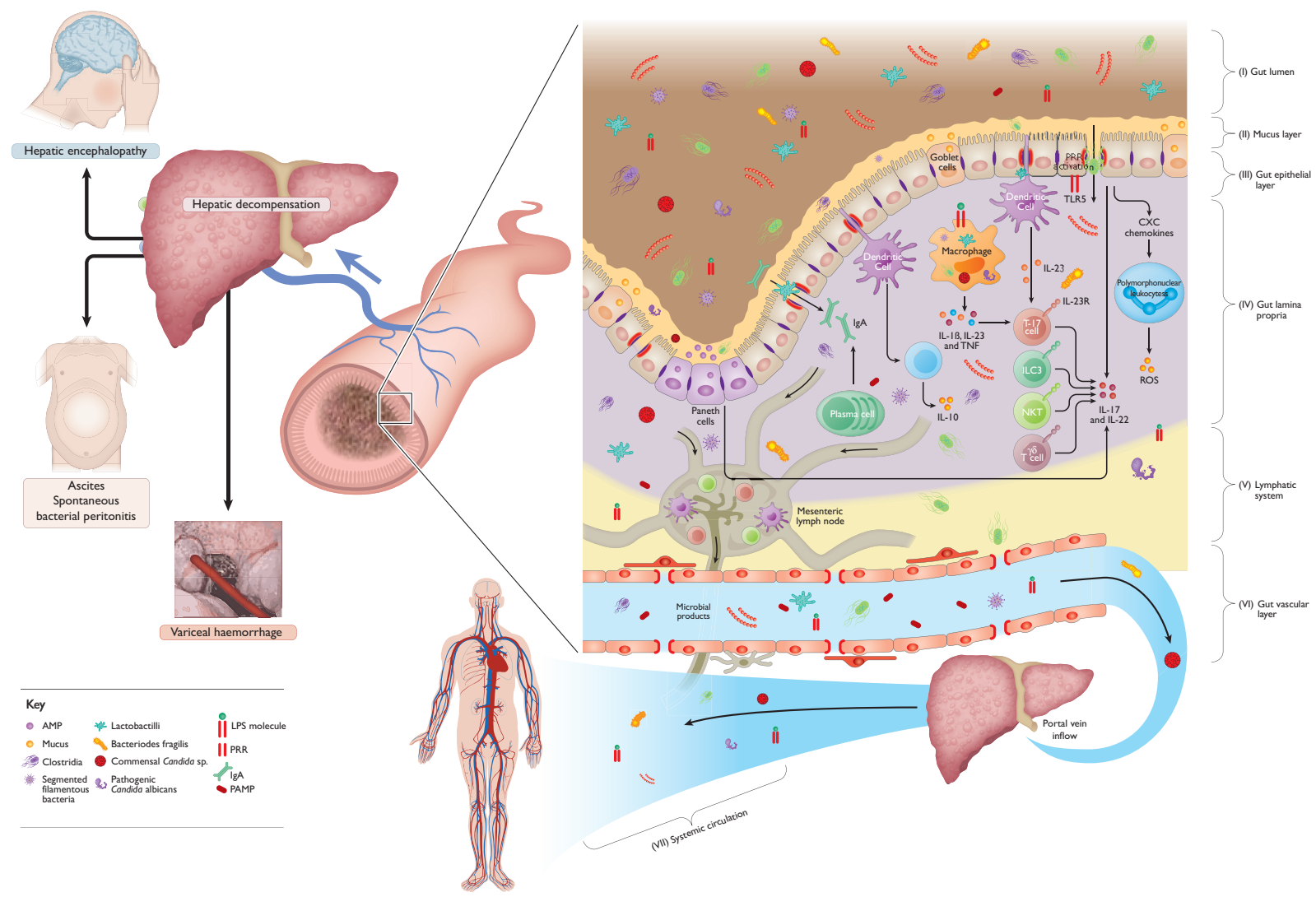

Fig 2. Relationship of the gut microbiome to other components of the intestinal barrier and gut-liver axis in cirrhosis. Box 1 gives further details on the role of the individual components and compartments of the intestinal barrier in cirrhosis. LPS = lipopolysaccharide; PAMP = pathogen-associated molecular patterns; $\mathrm{PRR}=$ pattern recognition receptor; $\mathrm{ROS}=$ reactive oxygen species. 
which drives inflammation and cirrhosis-associated immune dysfunction. ${ }^{24}$ Disrupted tight junction expression affecting the small intestines of cirrhotic patients has been shown to correlate with degree of endotoxaemia based on measurement of lipopolysaccharide (LPS), one of the prototypical PAMPs. ${ }^{25}$ The GVB has recently been described as a key layer of defence and is disrupted in non-alcoholic fatty liver disease (NAFLD), allowing translocation of bacteria and PAMPs into the circulation, as demonstrated by increased detection of the fenestrated marker PV1. ${ }^{26}$ As a consequence, the intestinal barrier in cirrhosis is often disrupted at multiple levels, propagating the pathological translocation of microbes and their inflammatory metabolites to the liver, where local hepatic inflammation is induced as well as to systemic sites via the lymphatic system (Fig 2; Box 1). The role of the gut microbiota in mediating and contributing to gut barrier disruption in cirrhosis remains to be determined. Recent studies have, however, begun to confirm the presence of gut inflammation in acutely decompensated cirrhosis by the evaluation of faecal cytokine profiles as well as other markers such as D-lactate, fatty acid-binding protein-2 and faecal calprotectin where intestinal dysbiosis is likely to play a causal role in affecting gut barrier integrity. ${ }^{27}$

Box 1. Individual components and compartments of the intestinal barrier and beyond involved in the gutliver axis in cirrhosis

I. Luminal content

> Microbiota dysbiosis with overgrowth of pathogens and depletion of commensal organisms

$>$ Overgrowth within small bowel with impaired gut mobility

$>$ Peturbed metabolic activity including bile acid profiles, short-chain fatty acids (SCFAs), lactate and tryptophan production

$>$ Increase in pathogen-associated molecular patterns (PAMPS) such as lipopolysaccharide (LPS), flagellin, peptidoglycan and unmethylated $\mathrm{CpG}$ motifs

II. Mucus layer

> Mucus layer in health prevents bacteria and their products from invading the microvillus environment

$>$ Mucus consists of glycoproteins secreted by goblet cells called mucins

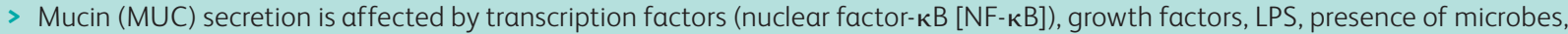
inflammatory cytokines

$>$ Animal models of cirrhosis show abnormal mucus production and function

III. Gut epithelial layer

$>$ Goblet and Paneth cell dysfunction, reduced mucin and antimicrobial proteins production

$>$ Ultrastructural changes: villous irregularity, increased epithelial cell space, tight junction compromise

$>$ Intracellular activation of pattern recognition receptors (PRRs)

> Translocation of viable bacteria and PAMPs

IV. Local mucosal immune responses

$>$ Increased neutrophil, macrophage and dendritic cell recruitment

$>$ Increased PRR activation

$>$ Enhanced pro-inflammatory mucosal cytokine release

$>$ Reduced anti-inflammatory metabolites

$>$ Enhanced mucosal priming of $\mathrm{T}_{H} \mathrm{I} / \mathrm{T}_{\mathrm{H}} 17$ cells

$>$ Reduced mucosal priming of T-regs

> Dysfunctional mucosal innate and adaptive immune cell responses

V. Gut-associated lymphoid tissue

> Comprises four lymphoid compartments: Peyer's patches, lamina propria lymphocytes, including dendritic cells (DCs), intraepithelial lymphocytes and mesenteric lymph nodes (MLNs)

> Microorganisms are transported by dendritic cells from intestines to MLNs via the lymphatic system

VI. Gut vascular layer

$>$ Consists of endothelial cells that express junctional proteins

> Enteric glial cells and pericytes together form the gut-vascular unit and are closely associated with endothelial cells

$>$ Enteric glial cells preserve integrity of epithelial and endothelial barriers through the release of S-nitroglutathione (GSNO)

$>$ Gut vascular barrier in cirrhosis is less able to control intestinal microbial products and antigens that translocate into the systemic circulation

VII. Systemic immune system

$>$ Heightened susceptibility to infection

> Immuno-paresis with systemic inflammation

> Defective innate and adaptive immune responses to PAMPs 


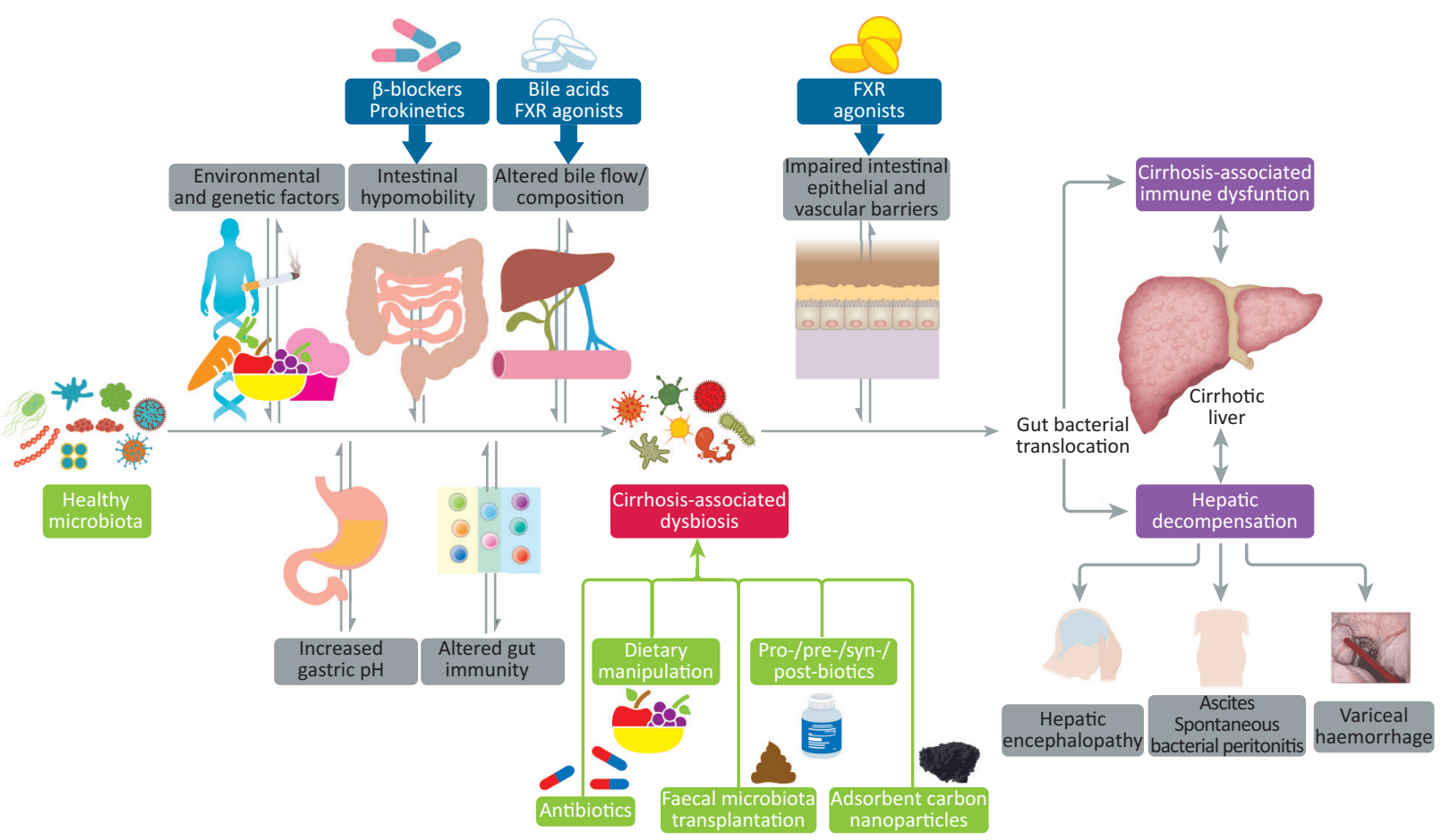

Fig 3. Therapeutic targets in and potential interventions to the gut-liver axis in cirrhosis.

\section{Manipulating the gut microbiota and gut-liver axis in cirrhosis}

Given that changes to the gut microbiota appear to be causal in propagating liver damage as well as hepatic decompensation in cirrhosis, the microbiota therefore rationally represents a therapeutically modifiable target. ${ }^{28,29}$ The gut microbiota can be manipulated by diet, drugs and pre-/probiotics as well as faecal microbiota transplantation or stool transplantation (FMT). These therapeutic approaches, many of which remain under investigation, are summarised in Fig 3.

Antibiotic therapy remains a cornerstone of treatment in cirrhosis, but has been associated with an increasing incidence of multidrug resistant infections in cirrhotic patients in recent studies from Europe and globally. ${ }^{30,31}$ Antimicrobial resistance (AMR) now poses a very major threat to cirrhotic patients, with the gut microbiota being a densely populated microbial ecosystem resident in the intestinal luminal environment that provides frequent opportunity for the horizontal transfer of resistance genes among microbes. This occurs through several different mechanisms including conjugation and transduction, with most AMR genes harboured by strictly anaerobic intestinal commensals. ${ }^{32}$ AMR is focusing efforts on investigating and developing effective alternative non-antibiotic based therapies for bowel decontamination designed to curtail PAMPs and bacterial translocation in cirrhosis. ${ }^{33}$ Rifaximin is a non-absorbable antibiotic licensed for the treatment of recurrent HE. Rifaximin's efficacy may be attributed to its impact on modulating the metabolic function of the gut microbiota rather than a change in relative bacterial abundance, although this is undergoing further evaluation in studies. $^{34}$

Prebiotics such pectin/inulin that nourish beneficial gut bacteria and probiotics, which are exogenously administered bacteria that confer a health benefit, have been used in cirrhosis, particularly for the treatment of $\mathrm{HE}$. A meta-analysis found that probiotics can decrease serum ammonia and endotoxin levels, improve minimal $\mathrm{HE}$, and prevent overt HE development in cirrhosis. ${ }^{35}$ Patients with acute alcoholic hepatitis (AAH) have been reported to have an over-representation of Enterococcus faecalis within their gut microbiota, with this species secreting a toxin called cytolysin which drives hepatocyte death and liver injury. ${ }^{36}$ Using humanised mice in the same study, bacteriophages, which are viruses that destroy bacteria with high specificity, were targeted against E faecalis; this resulted in decreased cytolysin in the liver and abolished ethanol-induced liver disease.

As a result of the success in manipulating the gut microbiome with single strains of bacteria using probiotics, interest has grown in replacing the entire dysbiotic gut microbiota with that of a healthy donor, using FMT. FMT has proven to be extremely successful in the treatment of recurrent Clostridium difficile infection. In cirrhosis, FMT has been studied in the treatment of $\mathrm{HE}$ where it was noted to reduce the number of $\mathrm{HE}$ episodes in the treatment group when given via enema after antibiotic pretreatment. ${ }^{37}$ FMT has also been used to treat steroid-ineligible $\mathrm{AAH}$ in India. ${ }^{38}$ Our group has recently completed a safety and feasibility study of FMT in advanced but stable cirrhosis (MELD score 10-16). ${ }^{39}$ This study showed that FMT was safe and feasible in this patient group, with a reduction in plasma ammonia levels in FMT treated patients persisting for up to 30 days after treatment. Ammonia is a microbial metabolite and has a central role in the pathogenesis of $\mathrm{HE}$, suggesting a potential role for the use of FMT in the management of this debilitating condition. FMT is currently only approved for use in clinical trials when prepared in a Medicines and Healthcare products Regulatory Agency licensed facility in the UK and the long-term effects of FMT are not yet 
known. A larger multicentre trial of encapsulated, lyophilized FMT (where the donor faeces are concentrated into ingestible capsules) is now planned in patients with decompensated cirrhosis in the UK with recruitment due to commence in 2021. Donors must be rigorously screened for potential pathogens to avoid the risk of transmission of infection to patients as occurred in the USA with an ESBL (extended spectrum beta lactamase)-producing bacteria traced back to infected FMT and which resulted in the death of one patient. $^{40}$

Other potential therapeutic agents that target the gut microbiota include non-selective beta-blockers (NSBBs), which have been shown to protect against SBP, potentially by reducing bacterial translocation by modulating gut motility and SIBO. ${ }^{41}$ Farnesoid $X$ receptor (FXR) agonists such as obeticholic acid and non-steroidal FXR agonists (PX2060) have been demonstrated to reduce gut permeability and portal pressures in a cirrhotic animal model, as well as reconstituting microbiota composition, restoring epithelial and vascular intestinal barrier function, improving intestinal innate defence mechanisms, reducing intestinal inflammation and decreasing bacterial translocation in experimental cirrhosis. ${ }^{42}$ Large-scale human studies are awaited. Synthetic carbons have been trialed as adsorbents to remove harmful bacterial metabolites as well as LPS from the gut. AST-120 reduced ammonia levels in cirrhotic rats, reducing brain water, but did not show a clinical benefit in humans. The CARBALIVE-SAFETY study (NCT03202498) is currently underway to assess the efficacy of Yaq-001 (a synthetic carbon) in humans, following successful animal studies. ${ }^{28}$

\section{Summary}

Cirrhotic patients exhibit intestinal dysbiosis which correlates with disease severity and endotoxaemia. Specific alterations in the gut microbiota have been associated with certain causes of cirrhosis and manifestations of hepatic decompensation, with research now focusing on establishing causality and confirming that these microbial changes are not simply due to bystander effects or epiphenomena. Manipulation of the gut microbiota is an increasingly attractive therapeutic target and can be achieved by several approaches which are non-antibiotic dependent in an era of rapidly increasing AMR incidence. There is emerging data on the utility of FMT as a therapeutic option in cirrhosis, as well as FXR agonists and NSBBs. Further studies are required to both mechanistically elucidate the pathophysiological role of the gut microbiota in cirrhosis, as well as the efficacy and safety of such targeted therapies on the gut-liver axis.

\section{In memoriam}

This article is dedicated to the late Prof Roger Williams CBE, who died recently after a short illness. He had a great interest in all aspects of the gut-liver axis in cirrhosis and for the potential for therapeutic interventions. He was, over the past 6 decades, a strong advocate for the role of the Royal College of Physicians in medical education. In recent years he was a highly valued mentor and supporter to Vishal Patel who, with many other academic hepatologists, continue in his legacy.

\section{References}

1 Marchesi JR, Ravel J. The vocabulary of microbiome research: a proposal. Microbiome 2015;3:31.
2 Human Microbiome Project Consortium. Structure, function and diversity of the healthy human microbiome. Nature 2012;486: 207-14.

3 Mullish BH, McDonald JAK, Pechlivanis A et al. Microbial bile salt hydrolases mediate the efficacy of faecal microbiota transplant in the treatment of recurrent Clostridioides difficile infection. Gut 2019;68:1791-800.

4 Macnaughtan J, Jalan R. Clinical and pathophysiological consequences of alterations in the microbiome in cirrhosis. Am J Gastroenterol 2015;110:1399-410.

5 Hooks KB, O'Malley MA. Dysbiosis and Its discontents. MBio 2017; 8:e01492-17.

6 Camilleri M, Madsen K, Spiller R, Greenwood-Van Meerveld B, Verne $\mathrm{GN}$. Intestinal barrier function in health and gastrointestinal disease. Neurogastroenterol Motil 2012;24:503-12.

7 Martens EC, Neumann M, Desai MS. Interactions of commensal and pathogenic microorganisms with the intestinal mucosal barrier. Nat Rev Microbiol 2018;16:457-70.

8 Chelakkot C, Ghim J, Ryu SH. Mechanisms regulating intestinal barrier integrity and its pathological implications. Exp Mol Med 2018;50:103.

9 Perez-Lopez A, Behnsen J, Nuccio SP, Raffatellu M. Mucosal immunity to pathogenic intestinal bacteria. Nat Rev Immunol 2016;16:135-48.

10 Peterson LW, Artis D. Intestinal epithelial cells: regulators of barrier function and immune homeostasis. Nat Rev Immunol 2014;14:141-53.

11 Spadoni I, Zagato E, Bertocchi A et al. A gut-vascular barrier controls the systemic dissemination of bacteria. Science 2015;350:830-4.

12 Allaire JM, Crowley SM, Law HT et al. The intestinal epithelium: central coordinator of mucosal immunity. Trends Immunol 2018; 39:677-96.

13 McDermott AJ, Huffnagle GB. The microbiome and regulation of mucosal immunity. Immunology 2014;142:24-31.

14 Qin N, Yang F, Li A et al. Alterations of the human gut microbiome in liver cirrhosis. Nature 2014;513:59-64.

15 Bajaj JS, Heuman DM, Hylemin PB, Sanyal AJ, White MB. Altered profile of human gut microbiome is associated with cirrhosis and its complications. J Hepatol 2014;60:940-7.

16 Yang $\mathrm{AM}$, Inamine $\mathrm{T}$, Hochrath $\mathrm{K}$ et al. Intestinal fungi contribute to development of alcoholic liver disease. J Clin Invest 2017;127: 2829-41.

17 Zhu L, Baker SS, Gill C et al. Characterization of gut microbiomes in nonalcoholic steatohepatitis (NASH) patients: a connection between endogenous alcohol and NASH. Hepatology 2013;57:601-9.

18 Kummen M, Holm K, Anmarkrud JA et al. The gut microbial profile in patients with primary sclerosing cholangitis is distinct from patients with ulcerative colitis without biliary disease and healthy controls. Gut 2016;66:611-9.

19 Pande C, Kumar A, Sarin SK. Small-intestinal bacterial overgrowth in cirrhosis is related to the severity of liver disease. Aliment Pharmacol Ther 2009;29:1273-81.

20 Chang CS, Chen GH, Lien HC, Yeh HZ. Small intestinal dysmotility and bacterial overgrowth in cirrhotic patients with spontaneous bacterial peritonitis. Hepatology 1998;28:1187-90.

21 Nardelli S, Gioia S, Ridola L et al. Proton pump inhibitors are associated with minimal and overt hepatic encephalopathy and increased mortality in patients with cirrhosis. Hepatology 2019;70:640-9.

22 Bajaj JS, Cox IJ, Betrapally NS et al. Systems biology analysis of omeprazole therapy in cirrhosis demonstrates significant shifts in gut microbiota composition and function. Am J Physiol Gastrointest Liver Physiol 2014;307:G951-7.

23 Bajaj JS, Acharya C, Fagan A et al. Proton pump inhibitor initiation and withdrawal affects gut microbiota and readmission risk in cirrhosis. Am J Gastroenterol 2018;113:1177-86.

24 Irvine KM, Ratnasekera I, Powell EE, Hume DA. Causes and consequences of innate immune dysfunction in cirrhosis. Front Immunol 2019;10:293.

25 Assimakopoulos SF, Tsamandas AC, Tsiaoussis GI et al. Altered intestinal tight junctions' expression in patients with liver cirrhosis: 
a pathogenetic mechanism of intestinal hyperpermeability. Eur ] Clin Invest 2012:42:439-46.

26 Mouries J, Brescia P, Silvestri A et al. Microbiota-driven gut vascular barrier disruption is a prerequisite for non-alcoholic steatohepatitis development. J Hepatol 2019;71:1216-28.

27 Riva A, Gray EH, Azarian S et al. Faecal cytokine profiling as a marke of intestinal inflammation in acutely decompensated cirrhosis. JHEP Reports 2020, in press (doi: 10.1016/j.jhepr.2020.100151).

28 Wiest R, Albillos A, Trauner M, Bajaj JS, Jalan R. Targeting the gut-liver axis in liver disease. J Hepatol 2017;67:1084-103.

29 Albillos A, Gottardi A, Rescigno M. The gut-liver axis in liver disease: pathophysiological basis for therapy. J Hepatol 2019;72:558-77.

30 Fernandez J, Prado V, Trebicka J et al. Multidrug-resistant bacterial infections in patients with decompensated cirrhosis and with acuteon-chronic liver failure in Europe. J Hepatol 2019;70:398-411.

31 Piano S, Singh V, Caraceni P et al. Epidemiology and effects of bacterial infections in patients with cirrhosis worldwide. Gastroenterology 2019;156:1368-80.

32 Ogilvie LA, Firouzmand S, Jones BV. Evolutionary, ecological and biotechnological perspectives on plasmids resident in the human gut mobile metagenome. Bioeng Bugs 2012;3:13-31.

33 Patel VC, Williams R. Antimicrobial resistance in chronic liver disease. Hepatol Int 2019;14:24-34.

34 Bajaj JS. Potential mechanisms of action of rifaximin in the management of hepatic encephalopathy and other complications of cirrhosis. Aliment Pharmacol Therapeut 2016;43:11-26.

35 Cao Q, Yu CB, Yang SG et al. Effect of probiotic treatment on cirrhotic patients with minimal hepatic encephalopathy: A metaanalysis. Hepatobiliary Pancreat Dis Int 2018;17:9-16.
36 Duan Y, Llorente C, Lang S et al. Bacteriophage targeting of gut bacterium attenuates alcoholic liver disease. Nature 2019;575: 505-11.

37 Bajaj JS, Kassam Z, Fagan A et al. Fecal microbiota transplant from a rational stool donor improves hepatic encephalopathy: a randomized clinical trial. Hepatology 2017:66:1727-38.

38 Philips CA, Pande A, Shasthry SM et al. Healthy donor fecal microbiota transplantation in steroid-ineligible severe alcoholic hepatitis: a pilot study. Clin Gastroenterol Hepatol 2017;15:600-2.

39 Woodhouse CA, Patel VC, Goldenberg S et al. PROFIT, a PROspective, randomised placebo controlled feasibility trial of Faecal mIcrobiota Transplantation in cirrhosis: study protocol for a single-blinded trial. BMJ Open 2019;9:e023518.

40 DeFilipp Z, Bloom PP, Torres Soto M et al. Drug-resistant E. coli bacteremia transmitted by fecal microbiota transplant. N Engl ] Med 2019:381:2043-50.

41 Senzolo M, Cholongitas E, Burra P et al. Beta-blockers protect against spontaneous bacterial peritonitis in cirrhotic patients: a meta-analysis. Liver Int 2009;29:1189-93.

42 Schwabl P, Hambruch E, Seeland BA et al. The FXR agonist PX20606 ameliorates portal hypertension by targeting vascular remodelling and sinusoidal dysfunction. J Hepatol 2017;66:724-33.

Address for correspondence: Dr Vishal Patel, Institute of Hepatology London (Foundation for Liver Research), 111 Coldharbour Lane, London SE5 9NT, UK.

Email: vish.patel@kcl.ac.uk 